hep-th/0212016

ITP-UH-26/02

\title{
Ghost Systems Revisited: Modified Virasoro Generators And Logarithmic Conformal Field Theories
}

\author{
Marco Krohn, Michael Flohr ${ }^{1}$ \\ Institute for Theoretical Physics \\ University of Hannover \\ Appelstraße 2, D-30167 Hannover, Germany
}

\begin{abstract}
We study the possibility of extending ghost systems with higher spin to a logarithmic conformal field theory. In particular we are interested in $c=-26$ which turns out to behave very differently to the already known $c=-2$ case. The energy momentum tensor cannot be built anymore by a combination of derivatives of generalized symplectic fermion fields. Moreover, the logarithmically extended theory is only consistent when considered on nontrivial Riemann surfaces. This results in a LCFT with some unexpected properties. For instance the Virasoro mode $L_{0}$ is diagonal and for certain values of the deformation parameters even the whole global conformal group is non-logarithmic.
\end{abstract}

\footnotetext{
${ }^{1}$ email: krohn@itp.uni-hannvoer.de, flohr@itp.uni-hannover.de
} 


\section{Introduction}

Only three years after Belavin, Polyakov and Zamolodchikov [1] started investigating conformal field theories in two dimensions it was noted by Knizhnik [2] that correlation functions may also exhibit logarithmic divergences. Six years later Gurarie [3] introduced the concept of a conformal field theory with logarithmic singularities: operator product expansions have the general form

$$
A(z) B(0)=z^{h_{C}-h_{B}-h_{A}} \sum_{n} \log ^{n}(x)\left\{C_{m-n}+\ldots\right\}
$$

where $C_{n}$ denotes the so-called logarithmic partners for $0 \leq n<m$ and $m$ gives the size of the Jordan-cell, i. e., the number of logarithmic partner fields which together with the primary field span a Jordan-cell for the zero mode $L_{0}$ of the Virasoro algebra. Thus, the basic feature of these so-called logarithmic conformal field theories (LCFTs) is that the representations of the chiral symmetry algebra may be indecomposable. It is worth noting that various aspects of LCFTs were noted in the literature before the work of Gurarie, e.g. $[4,5,6]$.

Logarithmic conformal field theories have a number of applications in very different fields, such as fractional quantum Hall effect, gravitational dressing, disorder, string theory and AdS/CFT to name only a few. For some recent reviews see $[7,8,9,10,11]$ and references therein.

Our own interest is related to the well-known $b$-c-ghost-system which is a quantum field theory of two anti-commuting fields $b, c$ with integer or halfinteger spins $\lambda$ and $1-\lambda$, respectively. The bosonic $\beta$ - $\gamma$-ghost-systems have recently been studied by $\mathrm{F}$. Lesage, P. Mathieu et al. [12]. It is conventional to call the spin $\lambda$ field $b(z)$ and the field with spin $1-\lambda$ then $c(z)$ with the choice $\lambda \geq 1-\lambda$. In this paper, we are interested in the integer spin case only. Of course correlators of the $b$ and $c$ fields do not show logarithmic behavior, but we claim that it is possible to enlarge the system to a logarithmic conformal field theory. To be more precise: we conjecture that each $b$-c-system (with central charge $\left.c_{b, c}=2\left(-1+6 \lambda-6 \lambda^{2}\right)\right)$ is in fact a subset of a larger logarithmic CFT with Jordan-cells of higher rank related to the spin $\lambda>0$. We will in detail study the case $c=-26$ which is the next integer spin case, $(\lambda, 1-\lambda)=$ $(2,-1)$, after the well-known $c=-2$ theory with $\operatorname{spin}(\lambda, 1-\lambda)=(1,0)$. Indeed we find a nontrivial indecomposable structure of the Virasoro modules 
which, however, is quite different from the Jordan-cell structure in the $c=-2$ system.

Our paper will proceed as follows:

In the next section we will briefly recall the main properties of the $c=-2$ LCFT. First we have a look at the construction via symplectic fermions. We then review an alternative approach [13] where the $c=-2$ LCFT is built via deformation of the energy momentum tensor.

The third section then deals with the generalization of these procedures to the ghost system with central charge $c=-26$. Firstly, we consider the zero mode structure of the fields by using a generalization of the symplectic fermion method. It turns out that the energy-momentum tensor cannot be constructed in a similar fashion as in the $c=-2$ case out of these fields, without running into severe difficulties. However, a generalization of the method of deformation is possible and yields a consistent representation of the Virasoro algebra. Thus, the two approaches are not equivalent in the $c=-26$ case.

Unfortunately, this Virasoro algebra does not act consistently on the Hilbert space of states of this theory. The reason for this is related to the origin of the logarithmic operators, which arise from operator product expansions of twist fields [14]. These twist fields exist whenever the theory is put on a nontrivial Riemann surface [2]. Thus, we investigate the theory on the simplest nontrivial Riemann surfaces, the hyper-elliptic ones, and find that the full theory features a consistent Virasoro algebra with the correct action on its space of states. Although this full theory turns out to be logarithmic, its structure is very different from the $c=-2$ case. For example, the zero mode of the Virasoro algebra, $L_{0}$, turns out to be diagonal, i. e., the Virasoro modules are not indecomposable with respect to $L_{0}$. However, other Virasoro modes definitely lead to indecomposable structures. The section concludes with building highest weight states for different conformal weights and discussing a suitable generalization of the Jordan-rank of the theory. 


\section{The $b, c$ ghost system as subset of logarith- mic $c=-2$ theory}

The conformal $b$ - $c$-system and the associated logarithmic so called $\theta-\bar{\theta}$-system for central charge $c=-2$ are well-known and have been intensely studied (see e.g. $[15,16,3,17,18,19]$ ). This is the reason for us having a closer look at this system again in the hope of learning how to build such logarithmic theories in general. In the case of $c=-2$ we will briefly repeat two different ways of building a LCFT: firstly via symplectic fermions [17, 20], and secondly by deforming the energy momentum tensor [13].

\section{1 $c=-2$ LCFT via Symplectic Fermions}

Following the approach described in [17] the $c=-2$ theory can be represented as a pair of ghost fields, or anti-commuting fields $\theta, \bar{\theta}$ of conformal weight $h=0$, with the free action [3]

$$
S=\int \mathrm{d}^{2} z \partial \theta \bar{\partial} \bar{\theta}
$$

(Note that $\theta, \bar{\theta}$ are not the complex conjugate of each other, but different fields.) As described in the above mentioned reference, the vacuum $|0\rangle$ is somewhat unusual, its norm is $\langle 0 \mid 0\rangle=0$, while the explicit insertion of the fields $\theta$ produces nonzero results, for instance $\langle\bar{\theta}(z) \theta(w)\rangle=1$. This property of the vacuum is believed to be typical for LCFTs.

Using the results given in [17] the mode expansion of the field $\theta$ (the analog holds for $\bar{\theta}$ ) is

$$
\theta(z)=\xi+\theta_{0} \log (z)+\sum_{n \neq 0} \theta_{n} z^{-n}
$$

where $\xi$ denotes the crucial zero modes and $n \in \mathbb{Z}$. The non-vanishing anticommutators $(n \in \mathbb{Z}, n \neq 0)$ are

$$
\left\{\theta_{n}, \bar{\theta}_{m}\right\}=\frac{1}{n} \delta_{n,-m}, \quad\left\{\xi, \bar{\theta}_{0}\right\}=1, \quad\left\{\theta_{0}, \bar{\xi}\right\}=-1,
$$

and together with the highest-weight relation

$$
\theta_{n}|0\rangle=0 \quad \forall n \geq 0,
$$


it is quite easy to see the logarithmic nature of the $\theta, \bar{\theta}$ system, for instance by calculating

$$
\langle\tilde{\mathbb{I}}(z) \tilde{\mathbb{I}}(w)\rangle=-2 \log (z-w)
$$

where $\tilde{\mathbb{I}}$ is defined as $\tilde{\mathbb{I}} \equiv-: \theta \bar{\theta}:$.

The stress energy tensor of the theory is

$$
T(z)=: \partial \theta \partial \bar{\theta}:
$$

and it is not hard to see that its expansion with $\tilde{\mathbb{I}}$ is indeed given by

$$
T(z) \tilde{\mathbb{I}}(w)=\frac{\mathbb{I}}{(z-w)^{2}}+\frac{\partial \tilde{\mathbb{I}}(w)}{z-w}+\ldots
$$

meaning that the operator $\tilde{\mathbb{I}}$ has conformal weight 0 . Also $\tilde{\mathbb{I}}$ is the logarithmic partner of $\mathbb{I}$, since $L_{0} \tilde{\mathbb{I}}=\mathbb{I}$. Thus, $\mathbb{I}$ and $\tilde{\mathbb{I}}$ span a Jordan-cell of rank two with respect to $L_{0}$. Indeed, the reader should convince herself that the action of $L_{0}$ cannot be diagonalized.

The most obvious differences between the $b$-c-system and the $\theta-\bar{\theta}$-system are

$$
\begin{array}{lcc}
\text { zero modes: } & \left(b_{0}, c_{0}\right) & \left(\bar{\theta}_{0}, \xi\right),\left(\theta_{0}, \bar{\xi}\right) \\
\text { conformal weights: } & h(b)=1, & h(\bar{\theta})=0, \\
& h(c)=0 & h(\theta)=0
\end{array}
$$

Therefore, in order to get from the $\theta-\bar{\theta}$-system to the $b$-c-system we have to reduce the number of zero mode pairs by one and also have to increase the conformal weight of one of the fields by one. This can easily be done by defining the transformation between $b, c$ and $\theta, \bar{\theta}$ in the following way:

$$
\begin{gathered}
b(z)=\sum_{n \in \mathbb{Z}} b_{n} z^{-n-1} \underset{b=\partial \bar{\theta}}{\stackrel{\bar{\theta}=\partial^{-1} b}{\longleftarrow}} \bar{\theta}(z)=\sum_{n \neq 0} \bar{\theta}_{n} z^{-n}+\bar{\theta}_{0} \log (z)+\bar{\xi} \\
c(z)=\sum_{n \in \mathbb{Z}} c_{n} z^{-n} \frac{\theta=c+\theta_{0} \log (z)}{\underset{c=\left.\theta\right|_{\theta_{0}=0}}{\longleftarrow}} \quad \theta(z)=\sum_{n \neq 0} \theta_{n} z^{-n}+\theta_{0} \log (z)+\xi
\end{gathered}
$$


While the derivative (respectively integration) gives the right transformation between $b$ and $\bar{\theta}$ we artificially have to add (respectively eliminate) a zero mode, $\theta_{0}$, to get the transformation between $c$ and $\theta$.

One might be tempted to use this method for constructing higher logarithmic CFTs, namely by putting the $b, c$ fields on equal footing by integrating the $b$ field $2 \lambda+1$ times where $\lambda>0$ denotes the conformal weight of the $b$ field. This integration leaves us with $2 \lambda+1$ new modes which then turn out to be one half of the total set of zero modes. The other half of the zero modes has to be added artificially in an analogous way, as for the $c$ field shown above. The latter are necessary as canonically conjugate partners for the zero modes arising as integration constants. Without these conjugate partners, the action of our zero modes would be trivial.

\section{$2.2 c=-2$ LCFT via logarithmic deformation}

As noted in the introduction of this section there is a different way to construct logarithmic extensions of conformal field theories as described in [13]. The idea of this method is to consider special deformations of the energy momentum tensor. One defines

$$
\tilde{T}:=T^{\mathrm{CFT}}+T^{\mathrm{impr}}
$$

where $T^{\text {impr }}$ denotes the so called "improvement term" which extends the CFT energy momentum tensor $T^{\mathrm{CFT}}$ in a way that the resulting stress tensor $\tilde{T}$ belongs to a logarithmic theory. Of course, the full stress energy tensor must still possess the correct operator product expansion with itself.

As is well-known the CFT stress tensor is given by

$$
T^{\mathrm{CFT}}=-\lambda: b(\partial c):+(1-\lambda):(\partial b) c:=-: b \partial c:
$$

for $\lambda=1$, which yields the $c=-2$ ghost-system. A careful consideration motivates the following ansatz for the improvement term:

$$
T^{\mathrm{impr}}=\frac{1}{z} \theta_{0} b(z)
$$

with $\theta_{0}$ being an additional zero mode. We have deliberately chosen to name this zero mode $\theta_{0}$ to make contact to the preceding approach via symplectic 
fermions. Indeed, the deformed energy momentum tensor can in this case be rewritten in a nicer form by applying a deformation to the fields as well:

$$
\begin{aligned}
& b(z) \longrightarrow \bar{\theta}(z)=\partial^{-1} b(z) \\
& c(z) \longrightarrow \theta(z)=c(z)+\theta_{0} \log (z)
\end{aligned}
$$

which leads to the well-known result (7). The theory with $c=-2$ is a bit special, because (as we will see later) it is not always possible to write the energy momentum tensor as a function of the new basic fields.

\section{A close look at $c=-26$}

Motivated by the success for the $c=-2$ system we are now going to construct a logarithmic conformal field theory for $c=-26$ which basically has the same properties as the $\theta, \bar{\theta}$ system in the $c=-2$ case. This construction process presumably does not only work for $c=-26$, but should work for any $b$-cghost-system.

One might be tempted to assume that the Jordan-cell of the LCFT for $c=$ -26 has a rank greater than two since this theory possesses a larger number of zero modes, i.e., modes which annihilate the vacuum to the left as well as to the right.

As we will see we do not find higher rank Jordan-blocks: in fact, the zero mode of the Virasoro algebra $L_{0}$ turns out to be perfectly well-defined without any Jordan structure at all. The nontrivial indecomposable structure of the Virasoro modules manifests itself in the action of the Virasoro modes $L_{n}, n \neq$ 0 . Therefore, we cannot speak of a rank of a Jordan-cell anymore. We will discuss later in which way the Virasoro modules are indeed indecomposable.

Investigating such systems is interesting for string theory. The calculation of string amplitudes makes use of the computation of $\lambda$-forms on nontrivial Riemann-surfaces. In a CFT approach these are the ghost systems. As Knizhnik [2] pointed out, nontrivial Riemann-surfaces, seen as a multisheeted covering of the complex plane, can be simulated by twist-fields inserted at the branch points. It has become clear by now that operator product expansions of such twist fields inevitable lead to logarithmic fields [21, 22]. Therefore, computation of string amplitudes automatically involves not only the $b, c$ system but its enlarged full LCFT. Also, there have been hints that LCFTs with 
higher rank Jordan-blocks play a role in the AdS/CFT correspondence [23]. Thus, it is important to learn more about LCFTs where the indecomposable structure is more involved than in the simple rank-two case. Even the simplest such higher-rank cases are very difficult to study, since the generic form of operator product expansion can only be fixed under quite restrictive assumptions [24].

\subsection{Generalizing symplectic fermions}

We now try to mimic what we did in the previous section, but this time for $c=-26$. Starting with the well-known (e.g. [25]) $b$-c-system for $c=$ -26 and by applying the same steps as we did for $c=-2$ we get a larger system. Unfortunately building a LCFT for $c=-26$ turns out to be more complicated than for the $c=-2$ case. Basically two obstacles are in the way of constructing a LCFT for $\lambda \geq 2$ :

1. The energy momentum tensor cannot be built by combining derivatives of the generalized symplectic fermion fields.

2. LCFT is intimately linked to twist fields arising from putting the CFT on a nontrivial Riemann-surfaces. The full theory is a tensor product of the CFTs for each covering sheet. We cannot neglect this fact.

In this sense $c=-2$ is special since the above mentioned problems do not show up (as we will explain later).

The $b$ - $c$-system for $c=-26$ is given by the following relations if we set $\lambda=2$ :

$$
\begin{aligned}
b(z)=\sum_{n} b_{n} z^{-n-\lambda}, & c(z)=\sum_{n} c_{n} z^{-n-(1-\lambda)} \\
\left\{c_{n}, b_{m}\right\}=\delta_{n+m, 0}, \quad\left\{c_{n}, c_{m}\right\}=\left\{b_{n}, b_{m}\right\}=0 & c_{n}|0\rangle=0 \quad \forall n \geq \lambda \\
b_{n}|0\rangle=0 \quad \forall n \geq-\lambda+1, \quad \forall n \geq . &
\end{aligned}
$$

Note that the $c=-26 b$-c-system comes with three pairs of zero modes, ${ }^{2}$ namely $\left(b_{i}, c_{i}\right)$ for $i=-1,0,1$. These modes are called zero modes for the

\footnotetext{
${ }^{2}$ If not explicitly stated otherwise the range of $i$ for the $c=-26$ system is $-1,0,1$
} 
following reason. As we can infer from the highest-weight conditions (18), the $b_{i}$ modes are annihilators to the left and to the right, while the $c_{i}$ modes are creators to the left and to the right. Thus, the $b_{i}$ are proper zero modes, and the $c_{-i}$ are their canonically conjugate partners. In the same way as we extended the $c=-2$ theory to a larger one by formal integration, we can try this for the $c=-26$ case by introducing the fields

$$
\begin{aligned}
\Lambda(z):= & \theta_{1} \log z+\theta_{0}(z \log z-z)+\theta_{-1} \frac{z^{2}}{2}\left(\log z-\frac{3}{2}\right) \\
& +\xi_{1}+\xi_{0} z+\xi_{-1} \frac{1}{2} z^{2}+\sum_{|n|>1} \theta_{n} \frac{z^{-n+1}}{-n+1} \\
\bar{\Lambda}(z):= & \bar{\theta}_{1} \log z+\bar{\theta}_{0}(z \log z-z)+\bar{\theta}_{-1} \frac{z^{2}}{2}\left(\log z-\frac{3}{2}\right) \\
& +\bar{\xi}_{1}+\bar{\xi}_{0} z+\bar{\xi}_{-1} \frac{1}{2} z^{2}+\sum_{|n|>1} \bar{\theta}_{n} \frac{z^{-n+1}}{-n+1} .
\end{aligned}
$$

The field $\bar{\Lambda}(z):=\partial^{-3} b$ has now the same conformal weight as its partner field $\Lambda(z):=c(z)+\sum_{i} f_{i}(z) \theta_{i}$. We call such pairs of anti-commuting fields of identical conformal weight generalized symplectic fermions. Note that the threefold-integration adds three new modes, $\xi_{i}, i=-1,0,1$, to the theory, which are (as we will see later) one half of the additional zero modes we have to add to the theory in order to make it logarithmic. The other half is artificially added in the $\Lambda$ field. Similar to the $c=-2$ case our new fields are now on equal footing $h(\Lambda)=h(\bar{\Lambda})=-1$.

Going from $\Lambda, \bar{\Lambda}$ back to $b, c$ of course requires removing these additional modes:

$$
\begin{aligned}
& b(z)=\partial^{3} \bar{\Lambda}(z) \\
& c(z)=\left.\Lambda\right|_{\theta_{i}=0} .
\end{aligned}
$$

The relations from the $b$-c-system can be translated to the new system and we find:

$$
\begin{array}{ll}
\left\{\theta_{n}, \bar{\theta}_{m}\right\}=-\frac{1}{n} \delta_{n,-m} & |n|,|m|>1 \\
\left\{\xi_{i}, \bar{\theta}_{-i}\right\}=(-1)^{i+1} & i=-1,0,1 .
\end{array}
$$


For the new modes we require the anti-commutation relations to be

$$
\left\{\bar{\xi}_{i}, \theta_{-i}\right\}:=(-1)^{i} \quad i=-1,0,1,
$$

which leads to the following OPEs

$$
\begin{aligned}
\Lambda(z) \bar{\Lambda}(w) & \sim \frac{1}{2}(z-w)^{2}\left[\frac{3}{4}-\log (z-w)\right] \\
\Lambda(z) \Lambda(w) & \sim \mathcal{O}(z-w) \\
\bar{\Lambda}(z) \bar{\Lambda}(w) & \sim \mathcal{O}(z-w) .
\end{aligned}
$$

The new modes indeed have the properties of zero modes, namely that all modes $\theta_{i}$ and $\bar{\theta}_{i}$ are annihilators to both sides, and the modes $\bar{\xi}_{-i}$ and $\xi_{-i}$ are their respective conjugate modes. Therefore, the extended theory also contains twice as many zero modes compared to the original $b$-c-ghost-system.

\subsection{Building the energy momentum tensor}

Having constructed fields which show logarithmic behavior leads us to the question how the energy momentum tensor for $c=-26$ looks like. Therefore, we look back to the $c=-2$ case in the hope to learn from this scenario. We

remember that for $\lambda=1$ respectively $c=-2$ simply plugging in the fields (9), (10) in the energy momentum tensor

$$
T=T[b, c]=-\lambda: b \partial c:+(1-\lambda):(\partial b) c: \quad .
$$

gives us the desired result (7). Unfortunately this does not work out in the same way for $c=-26$ and presumably neither for any other $\lambda \geq 2$.

The reason is obvious: $\Lambda$ appears plainly and as first derivative in the energy momentum tensor. Because of $\Lambda$ containing $z^{n} \log (z)$ terms this inevitable leads to logarithmic terms in the energy momentum tensor.

To find possible energy momentum tensors at all we use a different approach and consider possible extensions of the stress tensor on the mode level. This approach is motivated by the paper of Fjelstad, Fuchs et al.[13], but note that our deformation term is slightly more general and so is our result. The deformation term in [13] is always constructed from primary fields, which we do not assume here.

$$
\begin{aligned}
T^{\log }(z) & =T^{\mathrm{bc}}(z)+R(z) \\
& =\sum_{n} z^{-n-2}\left(L_{n}^{\mathrm{bc}}+R_{n}\right)
\end{aligned}
$$


where the modes $L_{n}^{\mathrm{bc}}$ are given by

$$
\begin{aligned}
L_{-2}^{\mathrm{bc}}= & -\sum_{l \neq-3, \ldots, 1} \frac{l(l+1)(l+4)}{l+3}: \bar{\theta}_{l} \theta_{-l-2}:-6: \bar{\theta}_{-3} \xi_{1}:-4: \bar{\theta}_{-2} \xi_{0}: \\
& -\frac{3}{2}: \bar{\theta}_{-1} \xi_{-1}:+\frac{4}{3}: \bar{\theta}_{0} \theta_{-2}:-\frac{5}{2}: \bar{\theta}_{1} \theta_{-3}: \\
L_{-1}^{\mathrm{bc}}= & -\sum_{l \neq-2, \ldots, 1} l(l+1): \bar{\theta}_{l} \theta_{-l-1}:-: \bar{\theta}_{-1} \xi_{0}:+: \bar{\theta}_{0} \xi_{-1}:-2: \bar{\theta}_{1} \theta_{-2}: \\
L_{0}^{\mathrm{bc}=} & -\sum_{l \neq-1, \ldots, 1} l^{2}: \bar{\theta}_{l} \theta_{-l}:-: \bar{\theta}_{1} \xi_{-1}:+: \bar{\theta}_{-1} \xi_{1}: \\
L_{1}^{\mathrm{bc}=} & \left.-\sum_{l \neq-1, \ldots, 2}(l-2)(l+1): \bar{\theta}_{l} \theta_{-l+1}:-3: \bar{\theta}_{-1} \theta_{2}:-2: \bar{\theta}_{0} \xi_{1}:-2: \bar{\theta}_{1} \xi_{d}(3)\right) \\
L_{2}^{\mathrm{bc}=} & -\sum_{l \neq-1, \ldots, 3} \frac{(l-4) l(l+1)}{l-1}: \bar{\theta}_{l} \theta_{-l+2}:-\frac{5}{2}: \bar{\theta}_{-1} \theta_{3}:+4: \bar{\theta}_{0} \theta_{2}: \\
& +6: \bar{\theta}_{1} \xi_{1}:+12: \bar{\theta}_{2} \xi_{0}:+6: \bar{\theta}_{3} \xi_{-1}:
\end{aligned}
$$

and $R_{n}$ denotes the extension which may contain the new deformation modes $\bar{\theta}_{i}, \xi_{i}$. The modes $L_{n}^{\log }:=L_{n}+R_{n}$ of course have to obey the Virasoro Algebra, which is a strong restriction. We get two different solutions, each coming with three possible deformations of the stress tensor:

$$
\begin{aligned}
R_{-2} & =6 A \theta_{1} \bar{\theta}_{-3}-4 B \theta_{0} \bar{\theta}_{-2}+\frac{3}{2} C \theta_{-1} \bar{\theta}_{-1} \\
R_{-1} & =-B \theta_{0} \bar{\theta}_{-1}-C \theta_{-1} \bar{\theta}_{0} \\
R_{0} & =-A \theta_{1} \bar{\theta}_{-1}+C \theta_{-1} \bar{\theta}_{1} \\
R_{1} & =2 A \theta_{1} \bar{\theta}_{0}+2 B \theta_{0} \bar{\theta}_{1} \\
R_{2} & =-6 A \theta_{1} \bar{\theta}_{1}+12 B \theta_{0} \bar{\theta}_{2}-6 C \theta_{-1} \bar{\theta}_{3} \\
R_{-2} & =6 A^{\prime} \theta_{1} \bar{\theta}_{-3}-B^{\prime} \theta_{-2} \bar{\xi}_{0}+\frac{3}{2} C^{\prime} \theta_{-1} \bar{\theta}_{-1} \\
R_{-1} & =-C^{\prime} \theta_{-1} \bar{\theta}_{0} \\
R_{0} & =-A^{\prime} \theta_{1} \bar{\theta}_{-1}+C^{\prime} \theta_{-1} \bar{\theta}_{1} \\
R_{1} & =2 A^{\prime} \theta_{1} \bar{\theta}_{0} \\
R_{2} & =-6 A^{\prime} \theta_{1} \bar{\theta}_{1}-3 B^{\prime} \theta_{2} \bar{\xi}_{0}-6 C^{\prime} \theta_{-1} \bar{\theta}_{3} .
\end{aligned}
$$

Testing the Virasoro Algebra with the above deformation terms is sufficient, since all higher modes can be derived with the help of the Virasoro Algebra. 
Two things are noteworthy: firstly, the second solution contains $\xi$ modes. This is a bit unexpected since $L_{n}^{\log }$ should, according to what we learned from the $c=-2$ theory, only lower the zero mode content and not increase it. Secondly, both solutions look very similar. Setting $B^{\prime}=0$ in the second solution, and thus eliminating the unwanted $\xi$-modes, would result in a special case $(B=0)$ of the first solution. As we will see later the second solution is indeed a special case of the first one. That is why we concentrate on the first solution for now.

The extensions can be written in a nicer way, making use of the $b$-field:

$$
T^{\log }(z)=T^{\mathrm{bc}}(z)+A \theta_{1} \frac{1}{z^{0}} \partial\left(z^{0} b\right)+B \theta_{0} \frac{1}{z^{1}} \partial\left(z^{2} b\right)+C \theta_{-1} \frac{1}{z^{2}} \partial\left(z^{4} b\right)
$$

which has a strikingly similarity with the energy momentum tensor deformations described by Fjelstad, Fuchs et al., but also has an important difference, namely the appearance of derivatives of the first order. The important point is that the deformations involve additional modes which are proper zero modes, i. e. annihilation operators to both sides. There are three possible "directions" to deform the energy momentum tensor, which matches exactly the number of zero modes of our system as we might have expected. In the $c=-2$ system only one such deformation was possible. There is another difference between $c=-2$ and $c=-26$ : while in the former theory it was possible to redefine the $b$ and $c$ fields (14), (15) in order to get an energy momentum tensor which consists of the new fields only, this is not possible in the latter case.

Demanding that the Virasoro modes satisfy the hermiticity condition $L_{n}^{\dagger}=$ $L_{-n}$ leads to a further restriction of the solution ${ }^{3}$ :

$$
A=C
$$

In the second solution, this requirement leads to the condition $A^{\prime}=C^{\prime}$.

\subsection{Fields on nontrivial Riemann Surfaces}

Up to now we have constructed fields $\Lambda, \bar{\Lambda}$ out of the $b$-c-system for $c=-26$ and we have found possible deformations of the energy-momentum tensor.

\footnotetext{
${ }^{3}$ Note that taking the adjoint of the modes can cause an additional constant, for instance $\xi_{1}^{\dagger}=\frac{1}{2} \xi_{-1}$, due to our normalization of the modes, which results from viewing them as integration constants.
} 
The Hilbert-space $\mathcal{H}^{\log }$ of the extended theory is an enlargement of the Hilbert-space of the $b$-c-system containing the additional zero modes $\bar{\xi}_{i}$.

This gives rise to another problem, namely that the constructed theory cannot be the full theory, because of $L_{0}^{\log }$ not being able to measure the conformal weight of all states contained in the Hilbert-space correctly. For instance $\left|\bar{\xi}_{-1}\right\rangle$ is surely an element of the Hilbert-space $\mathcal{H}^{\log }$, but $L_{0}^{\log }\left|\bar{\xi}_{-1}\right\rangle=0$ gives the wrong conformal weight.

This is an extremely interesting observation. The origin of logarithmic fields is tied to the existence of so-called pre-logarithmic primary fields, whose operator product expansions contain the logarithmic fields [14]. In fact, the first hint for the existence of the field $\tilde{\mathbb{I}}$ in the $c=-2$ theory comes from evaluating the four-point function of four $\mathbb{Z}_{2}$ twist fields $\mu$ of conformal weight $h=-1 / 8$, as has been observed in [3]. As a result, this four-point function contains the following two conformal blocks:

$$
\begin{aligned}
& \langle\mu(\infty) \mu(1) \mu(x) \mu(0)\rangle=[x(1-x)]^{\frac{1}{4}} F(x), \\
& F(x)= \begin{cases}{ }_{2} F_{1}\left(\frac{1}{2}, \frac{1}{2} ; 1 ; x\right), \\
{ }_{2} F_{1}\left(\frac{1}{2}, \frac{1}{2} ; 1 ; 1-x\right) & ={ }_{2} F_{1}\left(\frac{1}{2}, \frac{1}{2} ; 1 ; x\right) \log (x) \\
+ & \left.\frac{\partial}{\partial \epsilon} 3 F_{2}\left(\frac{1}{2}+\epsilon, \frac{1}{2}+\epsilon, 1 ; 1+\epsilon, 1+\epsilon ; x\right)\right|_{\epsilon=0} .\end{cases}
\end{aligned}
$$

In case of the ghost systems, these pre-logarithmic twist fields have a geometric meaning: these fields behave exactly as branch points of a ramified covering of the complex plane. For example, the above mentioned $\mathbb{Z}_{2}$ twist fields $\mu$ simulate the branch point of a hyper-elliptic surface in case of the $c=-2$ theory. Whenever all branch points have the same ramification number, say $n$, all monodromies around these points can be diagonalized simultaneously.

As Knizhnik [2] has shown, ghost systems on such $\mathbb{Z}_{n}$-symmetric Riemann surfaces can be dealt with by putting them on an $n$-fold sheeted covering of the complex plane where the branch points are represented by suitable constructed vertex operators. However, these vertex operators are twist fields, and thus may produce logarithmic divergences in their operator product expansions. Furthermore, to yield a local theory, we have to take the tensor product of the theories on all covering sheets.

The simplest such case is the hyper-elliptic one, since then automatically all branch points are of order two. This hyper-elliptic case is special since 
for the $c=-2$ theory, and only for this theory, one of the two copies of the conformal field theory decouples completely. This is a major difference of the $c=-2$ theory compared to other ghost systems, namely that it is possible to eliminate the theory on one of the two covering planes because after diagonalizing the monodromies the vertex operators associated to the branch cuts become trivial on one of the sheets.

Since this is a subtle point, we discuss it a bit more in detail: The twist field $\mu$ for a branch point on a hyper-elliptic surface for the $c=-2$ ghost system is actually given by $\mu(z)=V_{-1 / 2}(z) \otimes V_{0}(z)$, where $V_{q}(z)$ denotes a vertex operator with charge $q$ with respect to the ghost current $J=: b c$ : in a free field construction, and where we have indicated the composition of the twist field out of the two copies of the CFT. The conformal weight is, with $h(q)=\frac{1}{2} q(q+1)$, given by $h\left(-\frac{1}{2}\right)+h(0)=-\frac{1}{8}$ as it should be. The background charge at infinity is for both copies $q_{0}=-1 / 2$ such that the total sum of all charges in each copy must add up to $2 q_{0}=-1$. Looking at the four-point function mentioned above, we actually have to compute

$$
\begin{aligned}
\left\langle\mu\left(z_{1}\right) \mu\left(z_{2}\right) \mu\left(z_{3}\right) \mu\left(z_{4}\right)\right\rangle= & \left\langle V_{-1 / 2}\left(z_{1}\right) V_{-1 / 2}\left(z_{2}\right) V_{-1 / 2}\left(z_{3}\right) V_{-1 / 2}\left(z_{4}\right)\right\rangle \\
& \times\left\langle V_{0}\left(z_{1}\right) V_{0}\left(z_{2}\right) V_{0}\left(z_{3}\right) V_{0}\left(z_{4}\right)\right\rangle \\
= & \left\langle Q_{+1} V_{-1 / 2}\left(z_{1}\right) V_{-1 / 2}\left(z_{2}\right) V_{-1 / 2}\left(z_{3}\right) V_{-1 / 2}\left(z_{4}\right)\right\rangle \\
& \times\left\langle Q_{-1} V_{0}\left(z_{1}\right) V_{0}\left(z_{2}\right) V_{0}\left(z_{3}\right) V_{0}\left(z_{4}\right)\right\rangle,
\end{aligned}
$$

where we have indicated the necessary screening charges in the last step. Now, we can easily construct a screening current with charge $q=1$ since $V_{1}(z)$ has conformal weight $h(q)=\frac{1}{2} q(q+1)=1$ as we expect. Actually, $V_{1}(z)$ behaves essentially in the same way as the screening current, since $J(z) \mathrm{d} z=: b c:(z) \mathrm{d} z$ transforms exactly like a one-differential. Thus $Q_{+1}=$ $\oint \mathrm{d} z V_{1}(z)$. This factor of the four-point function yields then precisely the integral representation of the hyper-geometric function appearing in (50). The second factor of the four-point function is more tricky, since the field $V_{-1}$ has conformal weight $h=0$, thus cannot serve as screening current. However, a screening current with the correct properties can be constructed in the form $Q_{-1}=\oint \mathrm{d} z \oint \mathrm{d} z^{\prime} V_{1}(z) V_{-2}\left(z^{\prime}\right)$, since $V_{-2}$ also has conformal weight $h=1$. When inserting these two screening charges, one has to be careful with the choice of the contour for the integration. It turns out that the net result in the presence of nothing but four identity fields $V_{0}\left(z_{i}\right), i=1, \ldots, 4$, simply is the operator : $\phi V_{-1}:(0)$, where $\phi(z)$ is the free field used in the bosonization. Thus, we end up with the insertion of the logarithmic partner $\tilde{\mathbb{I}}(0)$ of the 
identity such that the second factor of (51) does not vanish identically, but yields simply a constant. Taken all together, we arrive at (50).

Repeating this computation for the $c=-26$ ghost system is a bit more involved. The twist fields for the hyper-elliptic case have now the composition $\mu(z)=V_{-1 / 2}(z) \otimes V_{-1}(z)$, such that the second factor is not merely the identity operator. The conformal weights are now given by $h(q)=\frac{1}{2} q(q+3)$ and the background charge at infinity is now $-3 / 2$. The twist field has therefore conformal weight $h_{\mu}=h\left(-\frac{1}{2}\right)+h(-1)=-5 / 8+(-1)=-13 / 8$. Thus, we have to satisfy

$$
\begin{aligned}
\left\langle\mu\left(z_{1}\right) \mu\left(z_{2}\right) \mu\left(z_{3}\right) \mu\left(z_{4}\right)\right\rangle= & \left\langle V_{-1 / 2}\left(z_{1}\right) V_{-1 / 2}\left(z_{2}\right) V_{-1 / 2}\left(z_{3}\right) V_{-1 / 2}\left(z_{4}\right)\right\rangle \\
& \times\left\langle V_{-1}\left(z_{1}\right) V_{-1}\left(z_{2}\right) V_{-1}\left(z_{3}\right) V_{-1}\left(z_{4}\right)\right\rangle \\
= & \left\langle Q_{-1} V_{-1 / 2}\left(z_{1}\right) V_{-1 / 2}\left(z_{2}\right) V_{-1 / 2}\left(z_{3}\right) V_{-1 / 2}\left(z_{4}\right)\right\rangle \\
& \times\left\langle Q_{+1} V_{-1}\left(z_{1}\right) V_{-1}\left(z_{2}\right) V_{-1}\left(z_{3}\right) V_{-1}\left(z_{4}\right)\right\rangle, \quad(52
\end{aligned}
$$

where we have again indicated the necessary screenings. Here, the second factor is easier, since the screening charge $Q_{+1}$ can always be taken as the contour integration of the ghost current $J_{+1}(z) \equiv J(z)=: b c:(z)$, since it transforms by construction as a one-differential. Moreover, all charges $q$ are always defined with respect to this ghost current. This is true independent of the value of the spin $\lambda$ of the ghost system considered. Thus, the screening charge $Q_{+1}$ is always easy to construct.

For the first factor, we have to use a modified version of the screening current, since the current $\tilde{J}(z)=: V_{1} V_{-2}:(z)$, although it has the correct conformal weight $h=1$ and is a local chiral field, does not yield the correct charge. It is merely an alternative representation of the screening current. Instead, we might use $J_{-1}(z)=\oint \mathrm{d} z^{\prime} V_{1}(z) V_{-2}\left(z^{\prime}\right)=\oint \mathrm{d} z^{\prime}\left(z-z^{\prime}\right)^{-2} V_{-1}\left(z^{\prime}\right)$. This current has the correct charge, but the wrong conformal weight $h=0$. We arrive thus at a similar situation as with the second factor in the $c=-2$ case, namely where the effect of screening is the insertion of a non-trivial $h=0$ field.

However, it is possible to construct a correct screening for the first factor by making use of the non-trivial $h=5$ field of charge $q=2$, which is part of the extended chiral symmetry algebra of the $c=-26$ ghost system. The correct screening charge reads then

$$
Q_{-1}=\oint \mathrm{d} u_{1} \oint \mathrm{d} u_{2} \oint \mathrm{d} u_{3} V_{-1}\left(u_{1}\right) V_{-2}\left(u_{2}\right) V_{2}\left(u_{3}\right)
$$


The integrand has total conformal weight $h=(5)+(-1)+(-1)=3$, which after three integrations yields a conformally invariant object.

The lengthy discussion shows the following: Evaluating the four-point function of four $\mathbb{Z}_{2}$ twist fields in the $c=-26$ case as in (52) yields an expression which will exhibit logarithmic singularities just as in the $c=-2$ case. Indeed, the second factor in the $c=-26$ case is again related to an integral representation of a hyper-geometric system, ${ }_{2} F_{1}(1,0 ; 0 ; x)={ }_{1} F_{0}(1 ; x)=$ $\int_{x_{0}}^{x} \mathrm{~d} u(1-u)^{-2}$. The first factor, however, is much more complicated since it involves a three-fold integration

$$
\oint \mathrm{d} u_{1} \oint \mathrm{d} u_{2} \oint \mathrm{d} u_{3} \frac{\left(u_{1}-u_{2}\right)^{2}}{\left(u_{1}-u_{3}\right)^{2}\left(u_{2}-u_{3}\right)^{4}} \prod_{i=1}^{4} \frac{\left(z_{i}-u_{1}\right)^{1 / 2}\left(z_{i}-u_{2}\right)^{1}}{\left(z_{i}-u_{3}\right)^{1}} .
$$

After bringing the four-point function (52) into standard form with $z_{1}=\infty$, $z_{2}=1, z_{3}=x, z_{4}=0$ with $x$ the crossing ratio, one of the three integrations can be performed and yields a Lauricella system of $D$-type (see for example $[26]$ ), which is a generalized hyper-geometric system of several variables:

$$
\begin{gathered}
\left\langle V_{-1 / 2}(\infty) V_{-1 / 2}(1) V_{-1 / 2}(x) V_{-1 / 2}(0)\right\rangle=\oint \mathrm{d} u_{2} \oint \mathrm{d} u_{3}\left(u_{2}-u_{3}\right)^{-4} \\
\times F_{D}^{(3)}\left(\frac{3}{2},-\frac{1}{2},-2,2 ; 3 ; x, u_{2}, u_{3}\right) \frac{u_{3}\left(1-u_{3}\right)\left(x-u_{3}\right)}{u_{2}\left(1-u_{2}\right)\left(x-u_{2}\right)}
\end{gathered}
$$

The system $F_{D}^{(3)}$ has several solutions depending on the choice of the integration contour, some of them exhibiting logarithms when expanded around $x=0$. This is similar to the ordinary hyper-geometric case where a logarithmic solution appears whenever $c$ in ${ }_{2} F_{1}(a, b ; c ; x)$ is an integer. In fact, $F_{D}^{(3)}\left(\frac{3}{2},-\frac{1}{2},-2,2 ; 3 ; x, u, u\right)={ }_{2} F_{1}\left(\frac{3}{2},-\frac{1}{2} ; 3 ; x\right)$, which is a hyper-geometric system with the two expansions

$$
\begin{aligned}
& y_{1}=\quad \sum_{n} \frac{\left(\frac{3}{2}\right)_{n}\left(-\frac{1}{2}\right)_{n}}{(3)_{n}(1)_{n}} x^{n} \\
& y_{2}=\log (x) \sum_{n} \frac{\left(\frac{3}{2}\right)_{n}\left(-\frac{1}{2}\right)_{n}}{(3)_{n}(1)_{n}} x^{n}+\sum_{n} \frac{\partial}{\partial \epsilon}\left(\frac{\left(\frac{3}{2}+\epsilon\right)_{n}\left(-\frac{1}{2}+\epsilon\right)_{n}}{(3+\epsilon)_{n}(1+\epsilon)_{n}}\right)_{\epsilon=0} x^{n}
\end{aligned}
$$

around $x=0$. The full computation of this four-point functions is beyond the scope of this paper. 
We note once more that looking at $n$-point functions of twist fields reveals whether we should expect logarithmic operators and thus indecomposable structures in our CFT or not. The logarithmic operators get exchanged in the internal channels of the $n$-point functions of twist fields due to degeneracies in the moduli space of the considered Riemann surface, if branch points run into each other. The present case, $c=-26$, clearly shows all signs to be a logarithmic CFT.

This discussion motivates, however, that our logarithmic deformation of the ghost system is related to the above mentioned situation on nontrivial Riemann surfaces. For the sake of simplicity, we concentrate again on the hyperelliptic case. Doing so, we now have two sets of modes $\left(\theta_{n}^{p}, \bar{\theta}_{n}^{p}, \xi_{i}^{p}, \bar{\xi}_{i}^{p}, n \in\right.$ $\mathbb{Z}, p=1,2)$ and also two sets of deformation parameters: $A_{1}, A_{2}, B_{1}, B_{2}$.

The easiest unification of both theories is given by defining the modes of the unified theory in the following way:

$$
L_{n}^{\text {tot }}:=L_{n}^{\log , 1}+L_{n}^{\log , 2}
$$

which means that we indeed take simply the tensor product of the two isomorphic conformal field theories. However this alone does not lead to a proper theory, since the new modes $L_{n}^{\text {tot }}$ do not satisfy the Virasoro algebra. To achieve the latter we have to identify the new modes, $\bar{\xi}_{i}, \theta_{i}$, on the one plane with the $\xi_{i}, \bar{\theta}_{i}$ modes on the other covering plane, by demanding

$$
\begin{aligned}
& \theta_{i}^{1} \sim \bar{\theta}_{i}^{2} \\
& \bar{\xi}_{i}^{1} \sim \xi_{i}^{2} .
\end{aligned}
$$

and analogously for $\theta_{i}^{2}$ and $\bar{\xi}_{i}^{2}$. Using up two more degrees of freedom by setting

$$
\begin{aligned}
& A:=A_{1}=-A_{2} \\
& B:=B_{1}=-B_{2}
\end{aligned}
$$

we get

$$
\left[L_{n}^{\log , 1}, L_{m}^{\log , 2}\right]=0
$$

and therefore $L_{n}^{\text {tot }}$ now not only fulfills the Virasoro algebra with total central charge $2 \cdot(-26)=-52$, but also acts correctly on the full space of states. 
It is worth mentioning that our construction automatically and naturally forces us to consider the (deformed) ghost system conformal field theory on a nontrivial Riemann surface. Moreover, we also have to slightly alter Knizhnik's prescription of constructing the full conformal field theory. A consistent Virasoro algebra with the correct action on the Hilbert space can only be obtained, if the two copies are not simply added, but only if the zero modes of the two conformal field theories are intermixed. This, in essence, encodes that the action of the monodromies cannot be fully diagonalized, leading to indecomposable structures in the conformal field theory. It is very interesting that for $c=-26$, and presumably for any other ghost system with $\lambda \neq 1$, the deformation of the Virasoro algebra inevitably leads us to consider such tensor products of these ghost systems, which do not factorize completely. As mentioned above, the $c=-2$ case appears now as particularly simple, since here the factorization of the full theory in two copies still almost holds. ${ }^{4}$ Thus, our enlarged theory has a nice and natural geometrical interpretation.

Applying the same steps to our second solution (43)-(47) gives:

$$
\begin{aligned}
& A^{\prime}:=A^{\prime}{ }_{1}=-A^{\prime}{ }_{2} \\
& C^{\prime}:=C^{\prime}{ }_{1}=-C^{\prime}{ }_{2} \\
& B^{\prime}:=B_{1}^{\prime}=B^{\prime}{ }_{2}=0 .
\end{aligned}
$$

This means that the second solution is already included in the first one $(B=0)$ and in particular the condition (63) enforces the elimination of the terms containing $\xi$ modes. Therefore, it is sufficient to investigate the first solution though we bear in mind that $B=0$ might be an interesting choice.

Retranslating the system to the familiar $b$-c-system using the choice above leads to

$$
\begin{aligned}
R_{-2}^{\text {tot }} & =-\frac{1}{2} A b_{-3}^{1} b_{1}^{2}-2 B b_{-2}^{1} b_{0}^{2}-3 A b_{-1}^{1} b_{-1}^{2}+\frac{1}{2} A b_{-3}^{2} b_{1}^{1}+2 B b_{-2}^{2} b_{0}^{1} \\
R_{-1}^{\text {tot }} & =-(A+B) b_{-1}^{1} b_{0}^{2}-(A+B) b_{0}^{1} b_{-1}^{2} \\
R_{0}^{\text {tot }} & =0 \\
R_{1}^{\text {tot }} & =(A+B) b_{0}^{1} b_{1}^{2}+(A+B) b_{1}^{1} b_{0}^{2} \\
R_{2}^{\text {tot }} & =3 A b_{1}^{1} b_{1}^{2}+2 B b_{2}^{1} b_{0}^{2}+\frac{1}{2} A b_{3}^{1} b_{-1}^{2}+2 B b_{0}^{1} b_{2}^{2}+\frac{1}{2} A b_{-1}^{1} b_{3}^{2} .
\end{aligned}
$$

\footnotetext{
${ }^{4}$ Of course, one should in principle also identify the additional zero mode for the deformation with the zero mode of the other copy of the conformal field theory for the other sheet.
} 
Therefore, our theory is diagonal with respect to $L_{0}^{\text {tot }}$ for arbitrary $A$ and $B$. Off-diagonal contributions appear in all different modes for almost all nontrivial choices of $A$ and $B$. The only nontrivial exception is $A=-B$ which eliminates all off-diagonal elements for $L_{-1}^{\text {tot }}$ and $L_{1}^{\text {tot }}$ thus leading to a theory which is as "little" as possible logarithmic, in the sense that the $\mathrm{SL}(2, \mathbb{C})$ global conformal group is not deformed at all. In particular the second solution (43)-(47) which narrowed down to the first one with $B=0$ comes for all nontrivial choices always with a deformation term. Note that there is no physical reason forcing this choice. ${ }^{5}$ For any nontrivial choice of $A$ and $B$ it is inevitable that deformations of higher modes $|n| \geq 2$ occur.

The next question is, what the highest weight states for our enlarged theory are, since these correspond to the primary fields. Leaving aside twisted sectors of the theory, we found the following highest weight states for $h=$ $-2,-1,0$ (note that there are no such states for $h=1,2$ and that all states for $h=-2$ are highest weight states).

$$
\begin{aligned}
h=-2: \quad & c_{1}^{1} c_{1}^{2}|0\rangle, c_{0}^{1} c_{1}^{1} c_{1}^{2}|0\rangle, c_{1}^{1} c_{0}^{2} c_{1}^{2}|0\rangle, c_{0}^{1} c_{1}^{1} c_{0}^{2} c_{1}^{2}|0\rangle \\
h=-1: \quad & c_{1}^{2}|0\rangle, c_{1}^{1}|0\rangle \\
& c_{0}^{1} c_{1}^{1}|0\rangle,\left(c_{0}^{1} c_{1}^{2}-c_{1}^{1} c_{0}^{2}\right)|0\rangle, c_{0}^{2} c_{1}^{2}|0\rangle \\
& \left(c_{0}^{1} c_{1}^{1} c_{0}^{2}-2 c_{-1}^{1} c_{1}^{1} c_{1}^{2}\right)|0\rangle,\left(c_{0}^{1} c_{0}^{2} c_{1}^{2}-2 c_{1}^{1} c_{-1}^{2} c_{1}^{2}\right)|0\rangle \\
& c_{-1}^{1} c_{0}^{1} c_{1}^{1} c_{1}^{2}|0\rangle,\left(c_{0}^{1} c_{1}^{1} c_{-1}^{2} c_{1}^{2}-c_{-1}^{1} c_{1}^{1} c_{0}^{2} c_{1}^{2}\right)|0\rangle, c_{1}^{1} c_{-1}^{2} c_{0}^{2} c_{1}^{2}|0\rangle, \\
& -(A+B) c_{-1}^{1} c_{1}^{1} c_{1}^{2}|0\rangle+c_{-1}^{1} c_{0}^{1} c_{1}^{1} c_{0}^{2} c_{1}^{2}|0\rangle, \\
& (A+B) c_{1}^{1} c_{-1}^{2} c_{1}^{2}|0\rangle+c_{0}^{1} c_{1}^{1} c_{-1}^{2} c_{0}^{2} c_{1}^{2}|0\rangle
\end{aligned}
$$

\footnotetext{
${ }^{5}$ If we want to keep the relations we already know from other LCFTs, then $A=-B$ is mandatory. In the so far known LCFTs, the Virasoro modes can be written in the form $L_{n}=z^{n}\left(z \partial_{i}+(n+1)\left(h_{i}+\delta_{h_{i}}\right)\right)$, see e.g. [16], such that $L_{-1}$ has no off-diagonal contribution, which one might expect for the generator of translations. This differs from the case considered here, where $L_{0}$ has no off-diagonal term. It follows then from the Virasoro algebra that $L_{-1}$ having no logarithmic contribution implies the same for $L_{1}$ and vice versa. The choice $A=-B$ reproduces this behavior.
} 


$$
\begin{aligned}
h=0: & |0\rangle \\
& c_{-1}^{1} c_{0}^{1} c_{1}^{1}|0\rangle, c_{-1}^{2} c_{0}^{2} c_{1}^{2}|0\rangle \\
& \left(c_{-1}^{1} c_{1}^{1} c_{0}^{2}-c_{-1}^{1} c_{0}^{1} c_{1}^{2}-c_{0}^{1} c_{1}^{1} c_{-1}^{2}\right)|0\rangle \\
& \left(c_{-1}^{1} c_{0}^{2} c_{1}^{2}+c_{1}^{1} c_{-1}^{2} c_{0}^{2}-c_{0}^{1} c_{-1}^{2} c_{1}^{2}\right)|0\rangle, \\
& \left(\frac{A^{2}}{4} c_{0}^{1} c_{0}^{2}+\frac{1}{2} A B c_{-1}^{1} c_{1}^{2}+\frac{1}{2} A B c_{1}^{1} c_{-1}^{2}+B c_{-1}^{1} c_{1}^{1} c_{-1}^{2} c_{1}^{2}\right. \\
& \left.+\frac{1}{2} A c_{-1}^{1} c_{0}^{1} c_{0}^{2} c_{1}^{2}+\frac{1}{2} A c_{0}^{1} c_{1}^{1} c_{-1}^{2} c_{0}^{2}+c_{-1}^{1} c_{0}^{1} c_{1}^{1} c_{-1}^{2} c_{0}^{2} c_{1}^{2}\right)|0\rangle
\end{aligned}
$$

As we noted above $L_{0}^{\text {tot }}$ is - in contrast to the $c=-2$ theory-diagonal. An operator for $c=-26$ which has similar properties as $L_{0}$ for $c=-2$ is $L_{-2}^{\text {tot }}$. Indeed, applying this operator generates off-diagonal terms as the following example shows

$$
L_{-2}^{\text {tot }}\left|c_{1}^{1} c_{1}^{2}\right\rangle=\left(c_{1}^{1} c_{-1}^{2}+c_{-1}^{1} c_{1}^{2}\right)|0\rangle-2\left(b_{-2}^{2} c_{0}^{2}+b_{-2}^{1} c_{0}^{1}\right)\left|c_{1}^{1} c_{1}^{2}\right\rangle+3 A|0\rangle .
$$

Applying $L_{-2}^{\text {tot }}$ a second time leads to further off-diagonal terms

$$
\begin{aligned}
\left(L_{-2}^{\mathrm{tot}}\right)^{2}= & -A\left(12 b_{-2}^{1} c_{0}^{1}+\frac{3}{2} b_{-3}^{1} c_{1}^{1}+12 b_{-2}^{2} c_{0}^{2}+\frac{3}{2} b_{-3}^{2} c_{1}^{2}\right)|0\rangle \\
& +8 B\left(b_{-2}^{1} c_{1}^{1} b_{-2}^{2} c_{1}^{2}\right)|0\rangle .
\end{aligned}
$$

While $A=-B$ in general makes the theory easier (by eliminating logarithmic contributions) this does not reduce the number of terms in this case.

If we multiply the deformation term with $q(A \rightarrow q A, B \rightarrow q B)$ then it is interesting to note that the power in $q$ does not go beyond a certain threshold if we consider $\left(L_{-2}^{\text {tot }}\right)^{m} \mid$ state $\rangle, m \in \mathbb{N}$. The reason for a threshold can be derived from the structure of the extension $R_{n}^{\text {tot }}$ and the states: each $R_{n}^{\text {tot }}$ contains at least one annihilator $b_{i}^{p}(p=1,2, i=-1,0,1)$ while the states are words in the conjugated modes, the creators, $c_{i}^{p}(p=1,2, i=-1,0,1)$ applied to the in-vacuum. By applying $R_{n}^{\text {tot }}$ the number of $c$ modes is reduced by one or two (or the term is eliminated) and most importantly there is no term in $L_{n}^{\text {tot }}$ which increases the number of $c$ modes again. Therefore, the maximum power in $q$ which theoretically can occur is 6 .

Our $c=-52$ theory comes, though logarithmic, with a non-logarithmic $L_{0}^{\text {tot }}$ which is a major difference to all LCFTs we know up to now. Because of $L_{0}^{\text {tot }}$ being trivial we obviously get no Jordan-cell or a Jordan-rank in the traditional sense. Nevertheless we have some properties which are the 
same in both types of LCFTs, the ones with and without logarithmic $L_{0}^{\mathrm{LCFT}}$. Remember that applying $L_{0}^{\mathrm{LCFT}}$ on a highest weight state $|h, k\rangle$ leads to an extra term $|h, k-1\rangle$ for $k>0$. Therefore, marking the logarithmic extension term with a $q$ leads to

$$
\left(L_{0}^{\log }\right)^{m}|h, k\rangle=q^{k}|h, 0\rangle+q^{k-1}(\ldots)+\ldots+h^{m}|h, k\rangle \quad, m>k
$$

where $k=0, \ldots$, jrk $\left(L_{0}^{\log }\right)-1$ and jrk denotes the rank of the Jordan-matrix. This means that the Jordan-rank can be found by applying $\left(L_{0}^{\mathrm{LCFT}}\right)^{m}$ for all $m \in \mathbb{N}$ on all $|h\rangle \in$ HWS (HWS denotes the set of all highest weight states). The highest occurring power in $q$ plus 1 defines the rank of the Jordan-cell. This motivates the following definition: writing $L_{n}^{\mathrm{LCFT}}=L_{n}^{\mathrm{CFT}}+q R_{n}$ where $R_{n}$ is the deformation term

$$
\operatorname{jrk}\left(L_{n}^{\log }\right):=\max \left\{k=\operatorname{deg}_{q}\left(\left(L_{0}^{\log }\right)^{m}|h\rangle\right):|h\rangle \in \mathrm{HWS}, m \in \mathbb{N}\right\} .
$$

where $\left(L_{0}^{\log }\right)^{m}|h\rangle$ is to be understood as a polynomial in $q$ after evaluation. The logarithmic behavior of this theory becomes (for $A=-B$ ) manifest in $L_{-2}^{\text {tot }}$. The Jordan-rank in the above defined sense of $L_{-2}^{\text {tot }}$ can easily be found by examining (67): each term contains at least one of the modes $b_{i}^{p}(p=1,2, i=-1,0,1)$. The remaining $b$ modes are of no interest since these are creators and the zero modes are mutually distinct in each of the terms. Therefore, the only states we are interested in are words in the letters $c_{-1}^{2}, c_{0}^{2}, c_{1}^{1} c_{1}^{2}, c_{-1}^{1}, c_{0}^{1}$. Looking at the highest weight states of conformal weight $h=0$ in eq. (72) shows that one highest weight state really contains a state which consists of all the above letters implying an upper bound

$$
\operatorname{jrk}\left(L_{-2}^{\mathrm{tot}}\right)=5
$$

up to accidental cancellations. Tedious and lengthy calculations reveal that the upper bound is satisfied. We note for completeness that for $B=0, A \neq 0$ the Jordan-rank is jrk $\left(L_{-2}^{\text {tot }}\right)=3$.

\section{Summary \& Conclusion}

The well-known $b$-c-system with central charge $c=-26$ can be enlarged to a logarithmic CFT. In some aspects the transition is similar to the $c=-2$ 
case, in others it is completely different: the energy momentum tensor cannot be built by combining derivatives of the generalized symplectic fermion fields and we also have to consider that it is not correct to neglect one half of the theory if we investigate it on hyper-elliptic Riemann surfaces. On the contrary, enlarging the $c=-26$ ghost system to a logarithmic theory makes it necessary to consider this theory on nontrivial Riemann surfaces. This is natural and consistent with our understanding of the geometrical origin of logarithmic fields from operator product expansions of twist fields which simulate branch points. On the other hand, it is surprising in so far as it is possible to consider the logarithmic extension of the better known $c=-2$ theory without the need of putting it on higher genus Riemann surfaces. As we have seen, this is impossible for $c=-26$. Due to the particular structure of the vertex operators which represent the branch points, we conjecture that logarithmic extensions of other ghost systems with $\lambda \neq 1,2$ are only possible when considered on $\mathbb{Z}_{n}$-symmetric Riemann surfaces.

We are confident that the presented construction scheme works not only for $c=-26$ but for all $b$-c-ghost-systems. The deformation term we used in order to obtain the new energy-momentum tensor is slightly more general than the deformation term discussed in the paper by Fjelstad, Fuchs et al. [13], but is naturally linked to the zero mode structure of the ghost systems. Thus, we expect that the spin $(\lambda, 1-\lambda)$ ghost system has generically $2 \lambda+1$ deformation directions which presumably get restricted due to hermiticity conditions and consistency requirements for the action of the deformed Virasoro algebra of the full theory on the Hilbert space of states.

The structure of the logarithmic $c=-26$ theory is very different from what one might have expected in analogy to the $c=-2$ case: $L_{0}^{\text {tot }}$ is not logarithmic at all. This is a completely new property of a LCFT. Furthermore the special choice of the deformation parameter $A=-B$ (see eq. (48)) leads to a theory where the whole global conformal group is non-logarithmic. This special property is not yet completely investigated. The logarithmic character of the theory becomes manifest in $L_{-2}^{\text {tot }}$ which shows similar indecomposable properties as $L_{0}$ in a standard LCFT. A generalization of the definition of the Jordan-rank has been given which we used to find that the Jordan-rank of $L_{-2}^{\text {tot }}$ is 5 for all nontrivial choices of $A$ and $B$. This should help in identifying the proper generalization of "logarithmic partners" to primary fields, which is left for future work. 


\section{Acknowledgment}

The research of M.F. is supported by the European Union network HPRNCT-2002-00325 and the research of M. F. and M. K. is supported by the string theory network (SPP no. 1096), Fl 259/2-1, of the Deutsche Forschungsgemeinschaft. We thank Sebastian Uhlmann and the participants of the workshop Non-Unitary \& Logarithmic Conformal Field Theory, which was held 10-14 June 2002 at the Institute des Hautes Études Scientifiques, Paris, France, for numerous discussions.

\section{References}

[1] A. A. Belavin, A. M. Polyakov, and A. B. Zamolodchikov, "Infinite conformal symmetry in two-dimensional quantum field theory," Nucl. Phys. B241 (1984) 333-380.

[2] V. G. Knizhnik, "Analytic Fields on Riemann surfaces II," Commun. Math. Phys. 112 (1987) 567-590.

[3] V. Gurarie, "Logarithmic operators in conformal field theory," Nucl. Phys. B410 (1993) 535-549, hep-th/9303160.

[4] L. Rozansky and H. Saleur, "S and T matrices for the superU(1,1) WZW model: Application to surgery and three manifolds invariants based on the Alexander-Conway polynomial," Nucl. Phys. B389 (1993) 365-423, hep-th/9203069.

[5] H. Saleur, "Polymers and percolation in two-dimensions and twisted N=2 supersymmetry," Nucl. Phys. B382 (1992) 486-531, hep-th/9111007.

[6] H. Saleur, "Geometrical lattice models $\mathrm{N}=2$ supersymmetric theories in two-dimensions," Nucl. Phys. B382 (1992) 532-560, hep-th/9111008.

[7] M. Flohr, "Bits and pieces in logarithmic conformal field theory," hep-th/0111228.

[8] M. R. Gaberdiel, "An introduction to conformal field theory," Rept. Prog. Phys. 63 (2000) 607-667, hep-th/9910156. 
[9] S. Moghimi-Araghi, S. Rouhani, and M. Saadat, "Use of nilpotent weights in logarithmic conformal field theories," hep-th/0201099.

[10] S. Kawai, "Logarithmic conformal field theory with boundary," hep-th/0204169.

[11] R. Rahimi Tabar, "Disordered systems and logarithmic conformal field theory," cond-mat/0111327.

[12] F. Lesage, P. Mathieu, J. Rasmussen, and H. Saleur, "The su(2)(-1/2) WZW model and the beta gamma system," hep-th/0207201.

[13] J. Fjelstad, J. Fuchs, S. Hwang, A. M. Semikhatov, and I. Y. Tipunin, "Logarithmic conformal field theories via logarithmic deformations," Nucl. Phys. B633 (2002) 379-413, hep-th/0201091.

[14] I. I. Kogan and A. Lewis, "Origin of logarithmic operators in conformal field theories," Nucl. Phys. B509 (1998) 687-704, hep-th/9705240.

[15] A. Cappelli, L. S. Georgiev, and I. T. Todorov, "A unified conformal field theory description of paired quantum Hall states," Commun. Math. Phys. 205 (1999) 657-689, hep-th/9810105.

[16] M. Flohr, "Null vectors in logarithmic conformal field theory," hep-th/0009137.

[17] V. Gurarie, M. Flohr, and C. Nayak, "The Haldane-Rezayi quantum Hall state and conformal field theory," Nucl. Phys. B498 (1997) 513-538, cond-mat/9701212.

[18] H. G. Kausch and G. M. T. Watts, "A Study of W algebras using Jacobi identities," Nucl. Phys. B354 (1991) 740-768.

[19] H. G. Kausch, "Curiosities at c=-2," hep-th/9510149.

[20] H. G. Kausch, "Symplectic fermions," Nucl. Phys. B583 (2000) 513-541, hep-th/0003029.

[21] M. R. Gaberdiel and H. G. Kausch, "A rational logarithmic conformal field theory," Phys. Lett. B386 (1996) 131-137, hep-th/9606050. 
[22] M. A. I. Flohr, "Logarithmic conformal field theory and Seiberg-Witten models," Phys. Lett. B444 (1998) 179-189, hep-th/9808169.

[23] G. Giribet, "Prelogarithmic operators and Jordan blocks in SL(2)k affine algebra," Mod. Phys. Lett. A16 (2001) 821-834, hep-th/0105248.

[24] M. Flohr, "Operator product expansion in logarithmic conformal field theory," Nucl. Phys. B634 (2002) 511-545, hep-th/0107242.

[25] S. V. Ketov, Conformal Field Theory. World Scientific, 1995.

[26] H. Exton, Multiple Hypergeometric Functions and Applications. Chichester: Ellis Horwood etc., 1976. 